\title{
Forms of the Solar Corona and their Origin.
}

\author{
By Dr. Wrlliam J. S. Lockyer, Norman Lockyer Observatory, Sidmouth.
}

$\mathbf{I}^{\mathrm{T}}$ T has long been known that the solar corona undergoes cyclical changes of form synchronising approximately with the eleven-year period of solar activity as determined by the variation of the spotted area on the solar surface from year to year.

These forms are so pronounced and distinctive that they can be classified into three main types,

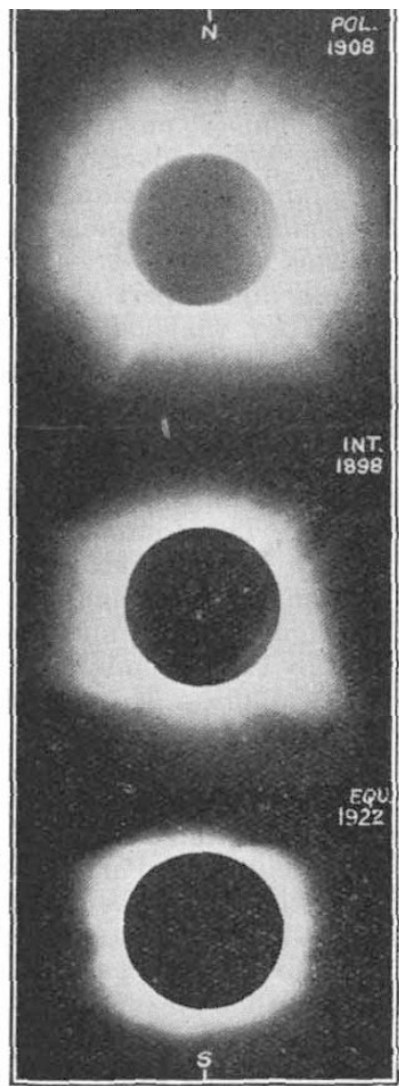

Fia. 1.-Types of solar corona. known as 'Polar', 'Intermediate', and 'Equatorial'. The characteristics of the types are as follows :

(1) The 'polar' type includes those irregular forms in which the streamers are situated all round the solar disc; the polar 'rifts' or 'plumes' in this type are conspicuous by their absence. This type is usually termed the 'maximum' or 'maximum sunspot' type.

(2) The 'equatorial' type is exhibited when the streamers are restricted to comparatively low solar latitudes, and the poles display the "polar rifts' to the best ad. vantage and have a large spread in latitude. This type is sometimes termed the 'minimum' or 'minimum sunspot' type, and also the "windvane' or 'fish-tail' form.

(3) The 'intermediate' type displays the streamers in mid-solar latitudes. The "polar rifts' are present, but not so extensive in latitude as in the 'equatorial' type. The streamers also approach nearer the polar regions than in the ' equatorial' type, while the equatorial extensions are not in such great evidence. This type is sometimes referred to as the 'square corona', and 'intermediate' between sunspot maximum and minimum type.

Illustrations of these types are shown in Fig. 1, and the coronas are so oriented that their north poles are situated at the top of each, and can therefore be compared directly with one another as regards the positions of the polar ' rifts' and 'steamers'. The corona at the top represents the 'polar' type (1908), and it will be seen that long streamers are situated in nearly all solar latitudes and the polar rifts are absent. The corona is very extensive all around the dark moon, owing to the sun being at its maximum state of activity.

The next lower figure represents the 'intermediate' stage (1898), the polar rifts becoming apparent. The corona takes a square form, the main streamers radiating more in mid latitudes. In the last form, that of the 'equatorial' type (1922), the polar rifts are well pronounced and the whole corona is not so extensive all round the disc. It is difficult, however, to show the extent of the equatorial streamers, as they are much fainter than usual and to some degree become lost in the reproductions.

Owing to these changes of form varying with the sunspot curve - that is, the 'polar' forms synchronising approximately with the maxima of spotted area, the ' equatorial' with the minima of spotted area, and the 'intermediate' with the intervals between these maxima and minima-all investiga. tors who have studied this subject have concluded that these changes of form were due to the sunspots themselves.

When we come to examine the positions of spots on the solar surface, it is found first of all that they are never near the poles at all. The highest latitude they ever attain is somewhere about $45^{\circ}$ north or south, and this occurs only at about the time when the spots are very small and the sun at a low state of activity. When, however, the sun is most active and the spotted area at a maximum, their mean latitude is only about $15^{\circ}$ north and south.

It will thus be seen that the presence of the spots themselves cannot possibly be the origin of the coronal streams at the poles of the sun, because at the epochs of the occurrence of the latter phenomena the spotted activity is at a maximum in latitude $15^{\circ}$, a very long way away from the poles. If, therefore, the spots do not cause the polar streamers, then there is no reason why they should account also for the streamers which occur in the spot zones. There must, therefore, be some other form of solar activity which gives rise to these brilliant streamers which can radiate from any latitude.

When the frequency of occurrence of solar prominences is studied closely it is found that, from year to year, they wax and wane, synchronising approximately with the variation of the sunspot areas. Thus it may be said that the curves representing prominence frequency and spotted area are very closely allied or similar, and if the changes in the coronal forms follow closely those of spotted area, they may equally be stated to follow also those of the prominence frequency.

Prof. Ludendorff, in 1929 , made a very detailed study of this subject of the cause of the changes of coronal form, and concluded that this change varies with the sunspot period. $\mathrm{He}$ did not add

No. 3236, Vor. 128] 
that it corresponds with the frequency of prominences as well, but if he had, his deduction would have been correct. The great advantage of accepting prominence action rather than spot action as the cause of the coronal streams is that prominences can occur in any latitude, even at the solar poles.

To illustrate the distribution in latitude of the prominences on the solar disc year by year, reference may be made to Fig. 2, where the latitudes ence zones from year to year, it will be seen that in both hemispheres there are nearly always two zones of prominences in action simultaneously.

That which may be termed the "low-latitude zone' commences two to three years after sunspot minimum, tends to move (like the spot zone) to lower latitudes, and disappears generally about two years before the next sunspot minimum. The other zone, which may be called the ' high-

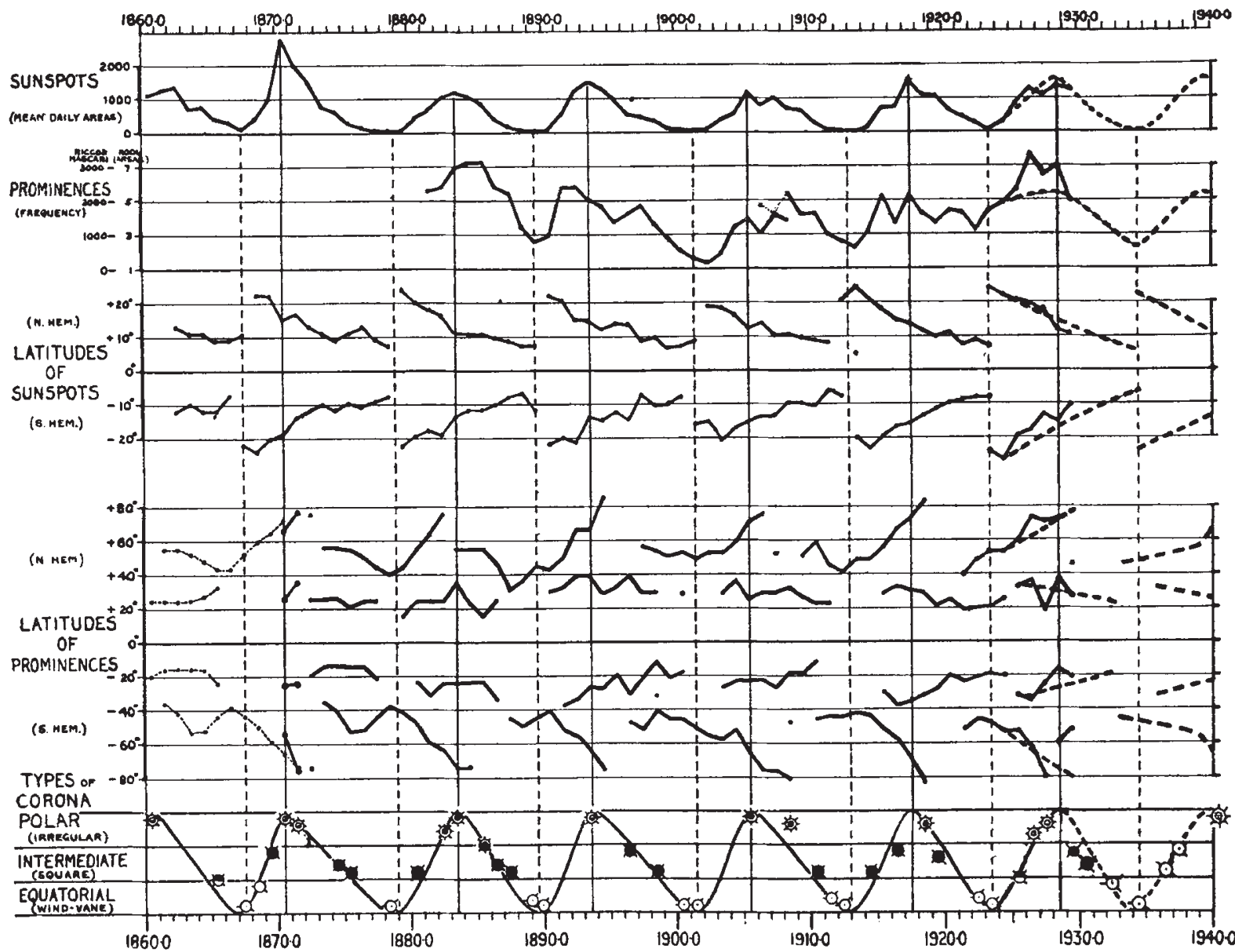

FIG. 2.-Periodicity of solar phenomena. Reproduced by courtesy of the Royal Astronomical Society from Mon. Not. R.A.S., vol. 91.

are given for each year from 1870 to 1929 for both hemispheres of the sun. In this figure the other solar changes already mentioned are also inserted, so that they can all be compared with one another on the same time scale. Thus, the first two curves show the strong similarity between the variations of the spotted areas and the prominence frequencies, and the approximate similarity of epochs of their maxima and minima. The third curve displays the change of latitude of the mean spotted area for each year for both hemispheres, demonstrating the movements towards the equator of the spot zones from a sunspot minimum to the following ninimum. The vertical continuous lines show the epochs of sunspot maximum, and the vertical broken lines those of sunspot minimum.

Coming now to the fourth series of curves, namely, those showing the latitudes of the promin- latitude zone', commences about three years after sunspot maximum, and moves towards the poles in each hemisphere, terminating generally very abruptly close to the poles about a year after sunspot maximum. At about the sunspot minimum there is usually a period of about two or three years when only one prominence zone is in operation, and that is the ' high-latitude zone', which is then situated in about latitude $45^{\circ}$ in each hemisphere.

When the sun is most active, as exhibited by either spots or prominences, the latitudes of the prominences exceed $60^{\circ}$ in both hemispheres in every case, except in the year 1928 , when it was at $60^{\circ}$ in the southern hemisphere only. Attention should specially be directed to the fact that the highest prominence latitudes do not necessarily occur at the sunspot maxima, but more generally about a year later, and in one instance, namely, in 1908, 
the highest latitude was attained in the southern hemisphere three years after the sunspot maximum.

Below these prominence latitude curves are shown the forms of the corona as observed at each eclipse. These are arranged in three horizontal strips, the uppermost one containing all the ' polar ' forms, the middle the 'intermediate', and the lowest the 'equatorial' forms. Each eclipse is placed in the year of its occurrence, according to the time scale given at the top and bottom of the figure. The continuous curve represents simply a smooth curve passing through the maxima and minima points, thus representing the sunspot curve.

An important comparison can now be made between these coronal types and the prominence latitudes. The first point to be noticed is that when the prominences exceed $60^{\circ}$ in latitude in either or both hemispheres, the corona is of the 'polar' type in every case. Here, therefore, we have a distant connexion between prominence activity and coronal streamers in the same high latitudes, where spots are never found.

There is also a very close association between the prominences and the other two coronal types. Thus the 'equatorial ' forms are recorded when the zone or zones of prominences are in their lowest latitudes; in general, this form is exhibited when there is only one prominence zone in operation. In nearly every case the ' intermediate' types are associated with two zones of prominences in each hemisphere.

Another interesting point arising out of these prominence latitude curves is that, according to the distribution of the prominences, there seems little doubt that the 'intermediate' type of corona takes on two forms, one as it occurs when the sun is increasing its activity and another when the sun's activity is on the wane. This difference is shown in the following table. In this the mean latitudes of both the high- and low-latitude prominence zones for each hemisphere, as shown in Fig. 2, are tabulated for each form of corona, and the 'intermediate' type is divided into two forms, 'intermediate up' and 'intermediate down'.

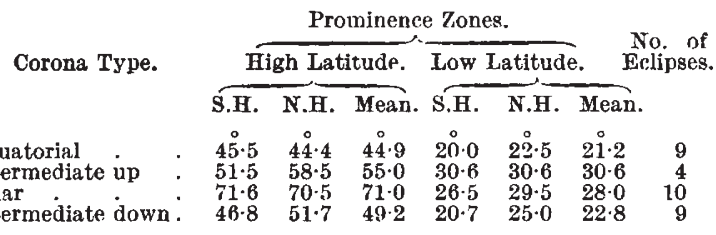

While there are only four eclipses of the ' up' type, but nine of the 'down', the latitudes of both the high- and low-latitude zones are greater in the former than in the latter.

Thus for the high-latitude zone the mean latitude for the 'up 'is nearly six degrees higher than that for the 'down', and for the low-latitude zone the ' up ' exceeds the ' down' by nearly eight degrees. This difference, which is in the same direction for both zones, seems to be sufficiently large to suggest that there may be a small change of type at the times of the rise and fall of solar activity.
It seems most probable that the form of the corona seen at any eclipse is not the immediate result of the presence of prominences at that particular moment. It seems more likely that the form is the result of long-continued action of the prominence zones, for weeks or maybe months, previous to the eclipse. If, for example, the prominences recorded on the sun for two or three months previous to an eclipse occurring were examined, it is most probable that the form of the corona could be well anticipated.

Using data selected from the years 1912, 1916, 1918, 1919, and 1923, since eclipses occurred in these years in which the coronal forms were recorded as of the equatorial, intermediate up, polar, intermediate down, and equatorial types respectively,

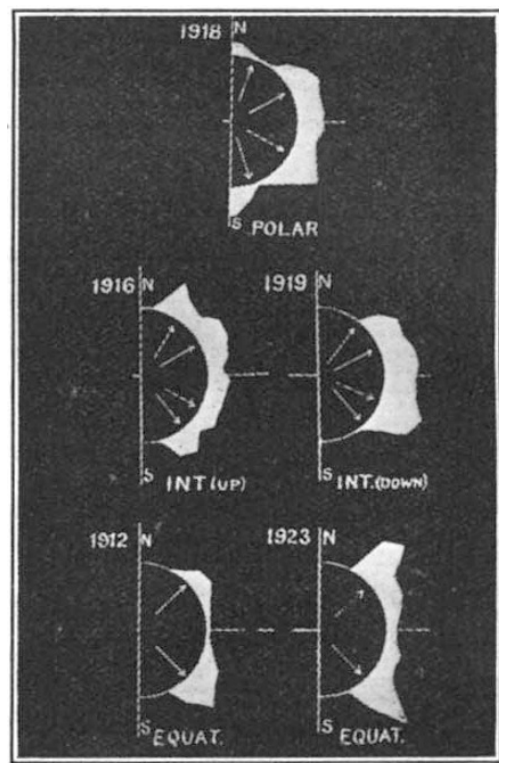

FIG. 3.-Coronal types in relation to latitudes of prominences. Reproduced by courtesy of the Royal Astronomical Society from Mon. Not. R.A.S., vol. 91 .

an attempt was made to see if they reflected the chief types of the corona.

These yearly mean prominence areas were then plotted for each ten degrees of latitude, as shown in Fig. 3, for each of the five years, the same scale being used throughout; this scale has, however, no special relation to the areas of the dark half suns, which are all of the same size. The sequence to be followed is from bottom left-hand side up to top right-hand side down.

It will be seen that the main features of the coronal types are all reflected in the prominence distribution in latitude, the high-latitude zones moving towards the poles simultaneously with the greater area of prominence activity, and reaching the poles at the epoch of the " polar' type of corona. The 'equatorial' type is conspicuous by having only one prominence zone in each hemisphere.

There is here a decided difference between the prominence distribution in the 'intermediate up. and the 'intermediate down', the latter, in this instance, conforming more to the equatorial type, 
disregarding the greater area of prominence activity there displayed.

The white arrows in each of the five half suns are the plotted positions of the latitudes of the high and low prominence zones extracted from the table previously given, and show the mean positions of these zones from all the eclipses of each type. It will be seen that, for this single series of years, the arrows conform very well to the actual positions of the prominence zones.

Another attempt was made, but this time only using data for the second half of the same years as indicated in Fig. 3. In this case the prominence areas displayed, quite astonishingly, practically the same distribution in latitude, and thus exhibited the same forms. These computations suggested very strongly that an actual corona is really the sum total of a prolonged prominence action previous to its appearance at an eclipse.

It will be noticed in Fig. 2 that from the year 1925 all the curves have been continued up to the year 1940 by broken lines. These were inserted in 1925 to attempt to forecast the changes in the different solar phenomena from year to year. Comparing those curves with those now available up to the year 1929, it will be seen that they conform fairly well with the actual facts. It was at that time considered probable that the high-latitude prominence zone would be still in evidence in both hemispheres in the year 1929 in about latitude $80^{\circ}$, and would therefore give rise to a 'polar' type of corona for that year. The latitude of this zone was, however, not so accommodating, but only actually reached $52^{\circ}$ in the southern hemisphere and $44^{\circ}$ in the northern hemisphere, so that an 'intermediate' type of corona should have been expected and was actually recorded.

In the case of the next few total solar eclipses following that of 1930 , namely, those for the years $1932,1934,1936,1937$, and 1940, the forecast curves suggest that the 1932 corona may be of the 'equatorial' or on the boundary between an 'intermediate' and 'equatorial' type ; that the 1934 corona will be of the pure ' equatorial' type ; that the coronas of 1936 and 1937 should display an 'intermediate' type; and, finally, that the 1940 corona should be of the 'polar' type, owing to the position of the high-latitude prominence zone, which should then be in about latitude $70^{\circ}$ in both hemispheres.

It is satisfactory to record that, since my first communication to the Royal Astronomical Society in 1903 , showing that the prominences are responsible for the varying shape of the solar corona, this view has been endorsed by J. Evershed in 1908 and by Prof. Osten Bergstrand in 1930.

\section{Gassendi and the Transit of Mercury.}

$\mathrm{T}$ THREE hundred years ago, in November and December 1631 , the first accurately predicted transits of both Mercury and Venus took place, but only the transit of Mercury was observed. Kepler many years before had stated that Mercury would cross the sun's disc on May 20, 1607, and carefully but vainly watched for it. With the completion of the Rudolphine Tables in 1627, he again took up the problem of determining the times of transit, and in a small tract published in 1629, entitled "Admonitio ad Astronomos rerumque celestium studiosos, de miris rarisque anni 1631 phaenomenis ...", announced that Mercury would pass over the sun's disc on Nov. 7, 1631, and Venus on Dec. 6, 1631 . He announced at the same time that there would not be another transit of Venus before 1761 , but in this he was in error.

Only one person was fortunate enough to observe the transit of Mercury predicted by Kepler, and this was Gassendi, who then, at the age of thirty-nine years, was already recognised as one of the foremost French philosophers. Some years afterwards, Gassendi became professor of mathematics at the Collège Royale in Paris and by his lectures and writings did much to stimulate an interest in science, but in 1631 he was provost of the Cathedral at Digne, near which he was born. He had been a precocious youth, lecturing at the age of sixteen and at the age of twenty-one becoming a professor of theology and philosophy at Aix. Among his contemporaries he counted Mydorge, Mersenne, and Descartes, while among his correspondents was Galileo. He was in Paris at the time of the transit and he gave an interesting account of his observations to Schickhard, professor of mathematics at Tübingen, a man as learned as himself.

Speaking of his observations, Gassendi said : " The crafty god had sought to deceive astronomers by passing over the sun a little earlier than was expected, and had drawn a veil of dark clouds over the earth in order to make his escape more effectual. But Apollo, acquainted with his knavish tricks from his infancy, would not allow him to pass altogether unnoticed. To be brief, I have been more fortunate than those hunters after Mercury who have sought the cunning god in the sun. I found him out, and saw him, where no one else had hitherto seen him.'

Gassendi had arranged to see the transit by admitting solar light into a dark room through a small hole and receiving the image of the sun upon a white screen marked with a circle divided into sixty parts. He placed an assistant in a room above, to observe the altitude of the sun with a $2 \mathrm{ft}$. quadrant, and to signal. Nov. 5 and Nov. 6 were both rainy and overcast and the morning of Nov. 7 broke with the weather still changeable. But fortune favoured him in the end and he was able to observe the planet some time before it passed off the disc about 10.30 A.M. He calculated that the transit had taken five hours and had occurred about four and three-quarter hours before the predicted time.

The second predicted transit of Mercury occurred on Nov. 3, 1651, and was observed at Surat in India by the young English astronomer, Jeremy Shakerley, who had gone to that country for the purpose; while the third transit recorded by astronomers was

No. 3236, VoL. 128j

@ 1931 Nature Publishing Group 